\title{
Don't run before you can walk
}

Licia Rivoltini, Andrea Marrari, Chiara Castelli, Sergio Villa and Riccardo Valdagni

We greatly appreciated the Nature Reviews Urology News \& Views article by Sharma and Logothetis (Prostate cancer: Combination of vaccine plus ipilimumab-safety and toxicity. Nat. Rev. Urol. 9, 302-303; 2012), ${ }^{1}$ fully agreeing with the authors' conclusions regarding the relevance of novel immunotherapeutics, but also sharing their concerns regarding the severe toxicity caused by cytotoxic T-lymphocyte protein 4 (CTLA4) blockade. In response to this article, we wish to offer a few additional insights into the use of immunotherapy for prostate cancer and some possible suggestions for maximizing the efficacy of ipilimumab, while maintaining an acceptable drug safety profile.

Although combination therapy (ipilimumab plus cancer vaccine) seems to have an adverse-effect profile comparable to that of ipilimumab alone, ${ }^{2,3}$ it is our opinion that combined treatment could introduce an additional level of unpredictable toxicity. In fact, vaccination could trigger bystander (self) crossreactive immune responses that might then be expanded by CTLA4-blockade, with an uncontrollable and unpredictable magnification of autoimmune reactions. Cellular vaccines based on allogenic prostate carcinoma cells might activate immune responses against normal epithelial antigens, which could exacerbate autoimmunity against normal mucosae and skin (upon amplification by ipilimumab). ${ }^{3}$ Viral-vector-based vaccines, on the other hand, could trigger $\mathrm{T}$ or $\mathrm{B}$ cells to crossreact with mimicking self-antigenic determinants (frequently identified in viral infections), leading to increased autoimmune toxicity. ${ }^{2,45}$ Thus, we speculate that ipilimumab should be combined with cancer vaccines that specifically target antigens expressed uniquely or prevalently by tumour cells. Whole proteins or long peptides derived from tumour-specific antigens-such as cancer-testis proteins ${ }^{6}$ or molecules linked to neoplastic transformation ${ }^{7,8}$-are appropriate for such a role.

We agree with the authors that the autoimmune toxicity induced by ipilimumab, with or without cancer vaccination, might be an acceptable adverse effect for many patients with advanced cancer, particularly as it can be reasonably controlled by corticosteroid therapy. However, cancer vaccines were primarily designed for patients with a small tumour burden and a conserved immune response (for example, to control minimal disease and prevent disease recurrence after surgery). ${ }^{9}$ Patients with prostate carcinoma in biochemical failure are ideal candidates for vaccination, as we recently observed during two phase II trials of peptide-based cancer vaccines at our institute (L. Rivoltini, unpublished data). ${ }^{10}$ Indeed, in our hands, these patients show very limited signs of immune system alteration (indicated by the low frequency and activity of regulatory $\mathrm{T}$ cells and myeloidderived suppressor cells in peripheral blood) and the ability to rapidly develop immunological responses to vaccination, with detectable clinical benefit (in terms of PSA kinetics). However, the issue of vaccine-related toxicity could be particularly relevant for these patients, especially when you consider their (relatively high) average age, the indolent nature of their disease, and the frequent need for long-term therapy.

One solution could be to use the vaccine to prime tumour-specific $\mathrm{T}$ and $\mathrm{B}$ cells and then administer ipilimumab at a low dose (or over a low-intensity schedule) in order to magnify the vaccine-induced immune response. This approach should limit the induction of self-reactive immune cells and, therefore, the full onset of autoimmunity. It is worth noting that antibodies that block other negative T-cell regulators (such as PD1 and PDL1) have also demonstrated high efficacy and acceptable toxicity profiles, ${ }^{11}$ and could soon be available for combination therapy with cancer vaccines. In the near future, urologists will be attempting to transfer data from more than two decades of experimental studies into the clinical setting. The next steps along this path should be considered carefully in order to maximize therapeutic success for patients, without substantially reducing their quality of life. With this aim in mind, we would like to refer to a highly relevant commentary by Drake entitled 'Immunotherapy for prostate cancer: Walk, don't run. ${ }^{12}$

Unit of Immunotherapy of Human Tumors (L. Rivoltini, C. Castelli), Prostate Cancer Programme (S. Villa, R. Valdagni), Adult Mesenchymal Tumor Medical Oncology Unit (A. Marrari), Fondazione IRCCS Istituto Nazionale dei Tumori, Via Venezian 1 , Milan 20133, Italy.

Correspondence to: L. Rivoltini

licia.rivoltini@istitutotumori.mi.it

Competing interests

The authors declare no competing interests.

1. Sharma, P. \& Logothetis, C. Prostate cancer: Combination of vaccine plus ipilimumabsafety and toxicity. Nat. Rev. Urol. 9, 302-303 (2012).

2. Madan, R. A. et al. Ipilimumab and a poxviral vaccine targeting prostate-specific antigen in metastatic castration-resistant prostate cancer: a phase I dose-escalation trial. Lancet Oncol. 13, 501-508 (2012).

3. van den Eertwegh, A. J. et al. Combined immunotherapy with granulocyte-macrophage colony-stimulating factor-transduced allogeneic prostate cancer cells and ipilimumab in patients with metastatic castration-resistant prostate cancer: a phase I dose-escalation trial. Lancet Oncol. 13, 509-517 (2012).

4. Blank, M., Asherson, R. A., Cervera, R. \& Shoenfeld, Y. Antiphospholipid syndrome infection origin. J. Clin. Immunol. 24, 12-23 (2004).

5. Loftus, D. J. et al. Identification of epitope mimics recognized by CTL reactive to the melanoma/melanocyte-derived peptide MART1(27-35). J. Exp. Med. 184, 647-657 (1996).

6. Caballero, O. L. \& Chen, Y. T. Cancer/testis (CT) antigens: potential targets for immunotherapy. Cancer Sci. 100, 2014-2021 (2009).

7. Straten, P. \& Andersen, M. H. The anti-apoptotic members of the $\mathrm{Bcl}-2$ family are attractive tumor-associated antigens. Oncotarget 1, 239-245 (2010).

8. Parmiani, G. et al. Universal and stemnessrelated tumor antigens: potential use in cancer immunotherapy. Clin. Cancer Res. 13, 5675-5679 (2007).

9. Kirkwood, J. M. et al. DERMA phase III trial of MAGE-A3 antigen-specific cancer immunotherapeutic ( $\mathrm{ASCl}$ ) as adjuvant therapy in patients with MAGE-A3-positive resected stage III melanoma. J. Clin. Oncol. 29, 2011 American Society of Clinical Oncology (ASCO) annual meeting suppl. abstract TPS232 (2011). 


\section{CORRESPONDENCE}

10. Marrari, A. et al. Vaccination therapy in prostate cancer. Cancer Immunol. Immunother. 56,

429-445 (2007)
11. Topalian, S. L. et al. Safety, activity, and immune correlates of anti-PD-1 antibody in cancer.

N. Engl. J. Med. 366, 2443-2454 (2012).
12. Drake, C. G. Immunotherapy for prostate cancer: walk, don't run. J. Clin. Oncol. 27, 4035-4037 (2009). 